\title{
OPEN Antimicrobial susceptibility of commensal Neisseria in a general population and men who have sex with men in Belgium
}

\author{
Jolein Gyonne Elise Laumen ${ }^{1,2,6}$, Christophe Van Dijck ${ }^{1,2,6}$, Saïd Abdellati ${ }^{1}$, \\ Irith De Baetselier ${ }^{1}$, Gabriela Serrano ${ }^{3}$, Sheeba Santhini Manoharan-Basil ${ }^{1}$, \\ Emmanuel Bottieau ${ }^{1}$, Delphine Martiny ${ }^{3,4}$ \& Chris Kenyon $[1,5 \square$
}

Non-pathogenic Neisseria are a reservoir of antimicrobial resistance genes for pathogenic Neisseria meningitidis and Neisseria gonorrhoeae. Men who have sex with men (MSM) are at risk of co-colonization with resistant non-pathogenic and pathogenic Neisseria. We assessed if the antimicrobial susceptibility of non-pathogenic Neisseria among MSM differs from a general population and if antimicrobial exposure impacts susceptibility. We recruited 96 participants at our center in Belgium: 32 employees, 32 MSM who did not use antibiotics in the previous 6 months, and 32 MSM who did. Oropharyngeal Neisseria were cultured and identified with MALDI-TOF-MS. Minimum inhibitory concentrations for azithromycin, ceftriaxone and ciprofloxacin were determined using E-tests ${ }^{\circledast}$ and compared between groups with non-parametric tests. Non-pathogenic Neisseria from employees as well as MSM were remarkably resistant. Those from MSM were significantly less susceptible than employees to azithromycin and ciprofloxacin $(p<0.0001, p<0.001)$, but not ceftriaxone $(p=0.3)$. Susceptibility did not differ significantly according to recent antimicrobial exposure in MSM. Surveilling antimicrobial susceptibility of non-pathogenic Neisseria may be a sensitive way to assess impact of antimicrobial exposure in a population. The high levels of antimicrobial resistance in this survey indicate that novel resistance determinants may be readily available for future transfer from non-pathogenic to pathogenic Neisseria.

Neisseria gonorrhoeae and N. meningitidis are becoming increasingly resistant to antimicrobials. For N. gonorrhoeae this concerns last-resort antimicrobials such as ceftriaxone and azithromycin ${ }^{1,2}$. Numerous studies have documented that for both species, much of this resistance has been acquired from the non-pathogenic Neisseria species that are a key component of a healthy oropharyngeal microbiome ${ }^{3-8}$. The most prominent genes involved in this transformation include penA, $m \operatorname{tr} C D E, r p l B, r p l D, r p l V$, parC, and $g y r A$. The acquisition of sections of these genes from non-pathogenic Neisseria has played an important role in the acquisition of penicillin, cephalosporin, macrolide, and/or fluoroquinolone resistance in N. meningitidis and N. gonorrhoeae $e^{5,9,10}$. Recent studies have established that uptake of DNA from non-pathogenic Neisseria was responsible for the majority of fluoroquinolone resistance in N. meningitidis and most azithromycin resistance in N. gonorrhoeae in Germany and the United States ${ }^{4,7,11}$. Non-pathogenic Neisseria have therefore gained interest as "canaries in the coalmine" for potential future resistance development in pathogenic Neisseria ${ }^{9,12,13}$.

Despite their importance as reservoirs of antimicrobial resistance (AMR), very few studies have explored the antimicrobial susceptibilities of contemporary non-pathogenic Neisseria. Studies of historical isolates found that non-pathogenic Neisseria were generally less susceptible to antimicrobials than pathogenic Neisseria $a^{9,13}$. In the last decade, however, few surveys have reported data on antimicrobial susceptibility of non-pathogenic Neisseria isolates. Two studies reported high minimum inhibitory concentrations (MICs) for macrolides, cephalosporins

\footnotetext{
${ }^{1}$ Department of Clinical Sciences, Institute of Tropical Medicine Antwerp, Nationalestraat 155, 2000 Antwerp, Belgium. ${ }^{2}$ Laboratory of Medical Microbiology, University of Antwerp, Wilrijk, Belgium. ${ }^{3}$ Department of Microbiology, Laboratoire Hospitalier Universitaire de Bruxelles, Pôle Hospitalier Universitaire de Bruxelles, Université Libre de Bruxelles, Brussels, Belgium. ${ }^{4}$ Faculté de Médecine et Pharmacie, Université de Mons, Mons, Belgium. ${ }^{5}$ Department of Medicine, University of Cape Town, Cape Town, South Africa. ${ }^{6}$ These authors contributed equally: Jolein Gyonne Elise Laumen and Christophe Van Dijck. ${ }^{凶}$ email: ckenyon@itg.be
} 
and fluoroquinolones among $N$. lactamica isolates from children in Japan and China in $2015^{14,15}$. One study found $93 \%$ fluoroquinolone resistance among commensal Neisseria from asymptomatic $N$. meningitidis carriers in China ${ }^{7}$. Two other studies were surveys among men who have sex with men (MSM) visiting a sexual health clinic in Vietnam in 2016 and Belgium in 2019 $9^{8,16,17}$. Both reported reduced susceptibility of non-pathogenic Neisseria to the antimicrobials currently used to treat gonorrhoea-azithromycin, and ceftriaxone. The high azithromycin and ceftriaxone MICs of non-pathogenic Neisseria among MSM is of particular concern as gonococcal AMR has frequently emerged in $\mathrm{MSM}^{18-20}$. MSM are also often co-colonised by N. meningitidis and $N$. gonorrhoeae in their pharyn $\mathrm{x}^{21-26}$.

Beyond these studies, very little is known about the epidemiology of antimicrobial susceptibilities in nonpathogenic Neisseria. In particular, little is known about their susceptibility in contemporary general adult populations.

It is not even known if the non-pathogenic Neisseria are more or less resistant in MSM than the general population and how MICs vary in relation to recent antimicrobial consumption.

Therefore, the aim of the current study was to compare the antimicrobial susceptibility of oropharyngeal Neisseria between MSM who recently used antimicrobials, MSM who did not, and employees of our institute as representatives of the general population in Belgium.

\section{Methods}

Survey population. This cross-sectional survey included 64 MSM and 32 employees.

The 64 MSM participated in a single centre randomized clinical trial (PReGo) at the Institute of Tropical Medicine (ITM) in Antwerp, Belgium in 2019-2020. PReGo was a placebo-controlled trial that assessed the efficacy of an antiseptic mouthwash (Listerine ${ }^{\mathrm{Tu}}$ ) to prevent STIs among $343 \mathrm{MSM}^{27}$. Taking HIV pre-exposure prophylaxis (PrEP) and having a history of gonorrhoea, chlamydia or syphilis in the previous two years was an inclusion criterium of that study. For the current survey, MSM were sampled at their first study visit, before administration of the PReGo study mouthwash. PReGo participants were enrolled into two groups, depending on their history of antimicrobial exposure.

Group I: MSM who recently used antimicrobials $(n=32)$. The first 32 PReGo participants who used at least one antimicrobial in the previous 6 months were included in this group.

Group II: MSM who did not recently use antimicrobials $(n=32)$. The first 32 PReGo participants who did not use any antimicrobial in the previous 6 months were included in this group.

Group III: Representatives of the general population: ITM employees who did not recently use antimicrobials $(n=32)$. In June 2020, ITM employees were invited to participate by posters and by word of mouth. Candidates who used an antimicrobial in the previous 6 months were excluded. The first 32 eligible employees (male or female) presenting to the study team were included in this survey.

Data collection and sampling procedure. All participants provided written informed consent prior to the collection of data and samples. Baseline characteristics were noted (including self-reported age, sex, antimicrobial use in the previous 6 months). Oropharyngeal samples were taken by a study physician who rubbed both tonsillar pillars and the posterior oropharynx with an ESwab ${ }^{\text {tw }}$ (COPAN Diagnostics Inc., Italy).

Sample processing. Culture and identification of Neisseria species. ESwabs ${ }^{\mathrm{Tm}}$ were inoculated onto Columbia Blood Agar and Modified Thayer-Martin Agar using the streak plate technique and incubated at 35$37^{\circ} \mathrm{C}$ and $5 \%$ carbon dioxide. Plates were examined after $48 \mathrm{~h}$ and Gram negative, oxidase positive colonies were selected, enriched and stored in Skim-milk at $-80^{\circ} \mathrm{C}$.

Isolates were identified to the species level using Matrix-Assisted Laser Desorption/Ionization-Time-of-Flight mass spectrometry (MALDI-TOF MS), on a MALDI Biotyper Sirius IVD system using the MBT Compass IVD software and library (Bruker Daltonics, Bremen, Germany). Briefly, each bacterial isolate was smeared twice on a polished steel target plate and then covered with $1 \mu \mathrm{L}$ of $\alpha$-cyano-4-hydroxycinnamic acid (CHCA) matrix solution. After drying, the target plate was loaded into the instrument. The spectra were acquired in linear mode in a mass range of $2-20 \mathrm{kDa}$ and subsequently compared to the library that included 9607 spectra at that time. Identification results were classified as reliable or unreliable according to recommended cut-off values of 1.7 and 2 for validated results for the genus and species levels, respectively. Only isolates belonging to the genus Neisseria were included in further analyses. Isolates identified as N. macacae were grouped into one category with $N$. mucosa, whereas isolates identified as $N$. perflava and $N$. flavescens were grouped into one category with N. subflava ${ }^{28}$.

Antimicrobial susceptibility determination. Minimum inhibitory concentrations (MICs) of Neisseria species to azithromycin, ceftriaxone, and ciprofloxacin were determined on GC agar plates using ETEST (bioMérieux Marcy-l'Étoile, France) incubated for $24 \mathrm{~h}$ at $36.5^{\circ} \mathrm{C}$ and $5-7 \% \mathrm{CO}_{2}$, and expressed in $\mathrm{mg} / \mathrm{L}$. Lack of bacterial growth during susceptibility testing resulted in missing values for that isolate.

Statistics. Neisseria prevalence. Prevalence was expressed as the proportion of participants from whom a certain species was isolated. Prevalence was compared between groups using Chi square tests. 


\begin{tabular}{|c|c|c|c|c|c|}
\hline & Overall $(n=96)$ & Employees $(n=32)$ & MSM who did not use antibiotics ( $n=32)$ & MSM who used antibiotics ( $n=32$ ) & $p$-value ${ }^{*}$ \\
\hline Age in years, median (IQR) & $35(35-47.5)$ & $45(35-55)$ & $45(35-55)$ & $39(35-45)$ & 0.21 \\
\hline Male sex, n (\%) & $74(77.1)$ & $10(31.3)$ & $32(100.0)$ & $32(100.0)$ & $<0.001$ \\
\hline $\begin{array}{l}\text { Antibiotic exposure in the previous } \\
6 \text { months, } n(\%)\end{array}$ & $32(33.3)$ & $0(0.0)$ & $0(0.0)$ & $32(100.0)$ & NA \\
\hline$\beta$-Lactams & $25(26.0)$ & NA & NA & $25(78.1)$ & NA \\
\hline Macrolides & $19(19.8)$ & & & $19(59.4)$ & \\
\hline Fluoroquinolones & $2(2.1)$ & & & $2(6.3)$ & \\
\hline Other & $8(8.3)$ & & & $8(25.0)$ & \\
\hline $\begin{array}{l}\text { Antibiotic exposure in the previous } \\
1 \text { month, } \mathrm{n}(\%)\end{array}$ & $7(7.3)$ & $0(0.0)$ & $0(0.0)$ & $7(21.9)$ & NA \\
\hline$\beta$-Lactams & $4(4.2)$ & NA & NA & $4(12.5)$ & NA \\
\hline Macrolides & $0(0.0)$ & & & $0(0.0)$ & \\
\hline Fluoroquinolones & $1(1.0)$ & & & $1(14.3)$ & \\
\hline Other & $2(2.1)$ & & & $2(6.3)$ & \\
\hline $\begin{array}{l}\text { Median number of casual sex partners in } \\
\text { the previous } 3 \text { months }\end{array}$ & NA & NA & $10.0(4.8-15.0)$ & $10.0(8.0-20.0)$ & 0.12 \\
\hline $\begin{array}{l}\text { Used condoms with }>75 \% \text { of casual anal } \\
\text { sex partners in the previous } 3 \text { months, } \\
n(\%)\end{array}$ & NA & NA & $9(28.1)$ & $2(6.5)^{\mathrm{a}}$ & 0.03 \\
\hline $\begin{array}{l}\text { Used a mouthwash in the previous } \\
1 \text { month, } \mathrm{n}(\%)\end{array}$ & 46 (47.9) & $15(46.9)$ & $12(37.5)$ & $19(59.4)$ & 0.22 \\
\hline
\end{tabular}

Table 1. Population characteristics. NA not applicable/not available. ${ }^{\star}$ Kruskal-Wallis rank sum test. ${ }^{a} 1$ missing value.

Neisseria species richness. Neisseria species richness was defined as the number of different non-pathogenic Neisseria species per participant. Species richness was reported as median (interquartile range) and compared between groups using Kruskal-Wallis rank sum tests. If no significant differences were observed between the two groups of MSM, their data were combined.

Antimicrobial susceptibility. To enable statistical testing, MICs above the maximum or below the minimum level of the ETEST strip were simplified as follows: azithromycin MIC $>256 \mathrm{mg} / \mathrm{L}$ was recoded as $512 \mathrm{mg} / \mathrm{L}$; ceftriaxone $\mathrm{MIC}<0.016 \mathrm{mg} / \mathrm{L}$ as $0.008 \mathrm{mg} / \mathrm{L}$; and ciprofloxacin MIC $>32 \mathrm{mg} / \mathrm{L}$ as $64 \mathrm{mg} / \mathrm{L}$. If multiple colonies of the same species were isolated from the same participant, we calculated the median MIC for that species per participant. MICs were reported as median (interquartile range) and compared between groups using KruskalWallis rank sum tests. If no significant differences were observed between the two groups of MSM, their data were combined. Pathogenic and non-pathogenic Neisseria were described and analysed separately, and subsequently stratified by species for species that were isolated at least once in each group.

In a sensitivity analysis, we used linear regression with geometric mean MIC as the outcome and two binary dependent variables: (a) being MSM/employee, and (b) antimicrobial exposure in the previous 6 months. The model was also adjusted for Neisseria species by the inclusion of a categorical variable.

All statistical analyses were performed with R version 4.0.5 (R Foundation for Statistical Computing, Vienna, Austria).

Ethics. Ethics approval was obtained from ITM's Institutional Review Board (1276/18 and 1351/20) and from the Ethics Committee of the University of Antwerp (19/06/058 and AB/ac/003).

The study was carried out according to the principles stated in the Declaration of Helsinki, all applicable regulations and according to the most recent GCP and GCLP guidelines. The Informed Consent Form (ICF) documents were designed in accordance with the requirements of the Helsinki Declaration (2013), the E6 ICH GCP Guidelines (2016) and the Belgian Law on Experiment on the Human Person (2004).

\section{Results}

The median age of the 96 participants was 35 (IQR 35-47.5) years (Table 1). Among the employees, two thirds were female. The MSM reported a high rate of partner change and a low rate of condom use, which is compatible with the high incidence of sexually transmitted infections in the PReGo study ${ }^{27}$. Of the 32 MSM who used antimicrobials in the previous 6 months, 14 (43.8\%) used only one class of antimicrobials, 14 (43.8\%) used two different classes of antimicrobials, and four (12.5\%) participants used three different classes of antimicrobials Supplementary information.

Neisseria prevalence. In total 207 Neisseria colonies were isolated, representing seven non-pathogenic and two pathogenic species (Table 2, Fig. 1). In descending order of prevalence, we isolated the non-pathogenic species $N$. subflava $(63 / 96,65.6 \%), N$. mucosa $(14 / 96,14.6 \%), N$. oralis $(8 / 96,8.3 \%), N$. cinerea $(3 / 96,3.1 \%), N$. 


\begin{tabular}{|c|c|c|c|c|}
\hline & $\begin{array}{l}\text { Prevalence }(\mathbf{n} / \mathrm{N}) \\
\text { Participants }(\%)\end{array}$ & $\begin{array}{l}\text { Azithromycin (mg/L) } \\
\text { Median (IQR) }\end{array}$ & $\begin{array}{l}\text { Ciprofloxacin (mg/L) } \\
\text { Median (IQR) }\end{array}$ & $\begin{array}{l}\text { Ceftriaxone }(\mathrm{mg} / \mathrm{L}) \\
\text { Median (IQR) }\end{array}$ \\
\hline Pathogenic Neisseria spp. & $27 / 96(28.1)$ & $0.5(0.4-0.9)$ & $0.004(0.003-0.006)$ & $<0.016(<0.016-<0.016)$ \\
\hline Neisseria meningitidis & $26 / 96(27.1)$ & $0.5(0.3-0.9)$ & $0.004(0.003-0.005)$ & $<0.016(<0.016-<0.016)$ \\
\hline Employees & $2 / 32(6.3)$ & $1.0(0.8-1.3)$ & $0.065(0.034-0.095)$ & $<0.016(<0.016-<0.016)$ \\
\hline $\begin{array}{l}\text { MSM who used } \mathrm{AB} \text { previous } \\
6 \text { months }\end{array}$ & $9 / 32(28.1)$ & $0.8(0.5-1.5)$ & $0.004(0.002-0.006)$ & $<0.016(<0.016-0.012)$ \\
\hline $\begin{array}{l}\text { MSM who used no } \mathrm{AB} \text { previous } \\
6 \text { months }\end{array}$ & $15 / 32(46.9)$ & $0.5(0.4-0.5)$ & $0.004(0.003-0.004)$ & $<0.016(<0.016-<0.016)$ \\
\hline Neisseria gonorrhoeae & $1 / 96(1.0)$ & 0.125 & 2.0 & $<0.016$ \\
\hline Employees & $0 / 32(0.0)$ & - & - & - \\
\hline $\begin{array}{l}\text { MSM who used } A B \text { previous } \\
6 \text { months }\end{array}$ & $0 / 32(0.0)$ & - & - & - \\
\hline $\begin{array}{l}\text { MSM who used no } \mathrm{AB} \text { previous } \\
6 \text { months }\end{array}$ & $1 / 32(3.1)$ & 0.125 & 2.0 & $<0.016$ \\
\hline Non-pathogenic Neisseria spp. & $65 / 96(67.7)$ & $3.0(2.0-7.5)$ & $0.032(0.016-0.25)$ & $0.047(0.029-0.064)$ \\
\hline Employees & $32 / 32(100.0)$ & $3.0(2.0-4.0)$ & $0.023(0.012-0.064)$ & $0.034(0.026-0.064)$ \\
\hline $\begin{array}{l}\text { MSM who used } \mathrm{AB} \text { previous } \\
6 \text { months }\end{array}$ & $19 / 32(59.4)$ & $16.0(3.0->256.0)$ & $0.250(0.141-0.500)$ & $0.047(0.032-0.094)$ \\
\hline $\begin{array}{l}\text { MSM who used no AB previous } \\
6 \text { months }\end{array}$ & $14 / 32(43.8)$ & $4.0(3.0-48.0)$ & $0.125(0.016-0.380)$ & $0.047(0.032-0.064)$ \\
\hline Neisseria subflava & $63 / 96(65.6)$ & $3.5(2.5-16.0)$ & $0.125(0.016-0.380)$ & $0.047(0.028-0.064)$ \\
\hline Employees & $31 / 32(96.9)$ & $3.0(2.3-4.0)$ & $0.032(0.016-0.197)$ & $0.035(0.028-0.052)$ \\
\hline $\begin{array}{l}\text { MSM who used AB previous } \\
6 \text { months }\end{array}$ & $13 / 32(40.6)$ & $288(3.5->256.0)$ & $0.380(0.190-0.500)$ & $0.064(0.032-0.064)$ \\
\hline $\begin{array}{l}\text { MSM who used no } \mathrm{AB} \text { previous } \\
6 \text { months }\end{array}$ & $19 / 32(59.4)$ & $4.0(3.3-72.0)$ & $0.125(0.022-0.380)$ & $0.047(0.028-0.126)$ \\
\hline Neisseria mucosa & $14 / 96(14.6)$ & $3.5(2.3-5.5)$ & $0.016(0.013-0.030)$ & $0.040(0.032-0.064)$ \\
\hline Employees & $8 / 32(25.0)$ & $3.5(2.8-4.5)$ & $0.017(0.011-0.025)$ & $0.040(0.032-0.072)$ \\
\hline $\begin{array}{l}\text { MSM who used AB previous } \\
6 \text { months }\end{array}$ & $4 / 32(12.5)$ & $3.5(2.8-6.3)$ & $0.133(0.015-1.688)$ & $0.040(0.032-0.051)$ \\
\hline $\begin{array}{l}\text { MSM who used no } \mathrm{AB} \text { previous } \\
6 \text { months }\end{array}$ & $2 / 32(6.3)$ & $12.6(6.9-18.3)$ & $0.016(0.016-0.016)$ & $0.063(0.048-0.079)$ \\
\hline Neisseria oralis & $8 / 96(8.3)$ & $2.0(1.9-3.1)$ & $0.015(0.012-0.018)$ & $0.056(0.032-0.064)$ \\
\hline Employees & $8 / 32(25.0)$ & $2.0(1.0-3.1)$ & $0.015(0.012-0.018)$ & $0.056(0.032-0.064)$ \\
\hline $\begin{array}{l}\text { MSM who used } \mathrm{AB} \text { previous } \\
6 \text { months }\end{array}$ & $0 / 32(0.0)$ & - & - & - \\
\hline $\begin{array}{l}\text { MSM who used no AB previous } \\
6 \text { months }\end{array}$ & $0 / 32(0.0)$ & - & - & - \\
\hline Neisseria cinerea & $3 / 96(3.1)$ & $2.0(1.5-15.0)$ & $0.012(0.009-0.022)$ & $<0.016(<0.016-<0.016)$ \\
\hline Employees & $3 / 32(9.4)$ & $2.0(1.5-15.0)$ & $0.012(0.009-0.022)$ & $<0.016(<0.016-<0.016)$ \\
\hline $\begin{array}{l}\text { MSM who used } \mathrm{AB} \text { previous } \\
6 \text { months }\end{array}$ & $0 / 32(0.0)$ & - & - & - \\
\hline $\begin{array}{l}\text { MSM who used no AB previous } \\
6 \text { months }\end{array}$ & $0 / 32(0.0)$ & - & - & - \\
\hline Neisseria elongata & $3 / 96(3.1)$ & $0.5(0.4-0.6)$ & $0.004(0.004-0.014)$ & $0.047(0.035-0.119)$ \\
\hline Employees & $3 / 32(9.4)$ & $0.5(0.4-0.6)$ & $0.004(0.004-0.014)$ & $0.047(0.035-0.119)$ \\
\hline $\begin{array}{l}\text { MSM who used AB previous } \\
6 \text { months }\end{array}$ & $0 / 32(0.0)$ & - & - & - \\
\hline $\begin{array}{l}\text { MSM who used no } \mathrm{AB} \text { previous } \\
6 \text { months }\end{array}$ & $0 / 32(0.0)$ & - & - & - \\
\hline Neisseria lactamica & $2 / 96(2.1)$ & $1.5(1.3-1.8)$ & $0.127(0.096-0.159)$ & $<0.016(<0.016-<0.016)$ \\
\hline Employees & $2 / 32(6.3)$ & $1.5(1.3-1.8)$ & $0.127(0.096-0.159)$ & $<0.016(<0.016-<0.016)$ \\
\hline $\begin{array}{l}\text { MSM who used } \mathrm{AB} \text { previous } \\
6 \text { months }\end{array}$ & $0 / 32(0.0)$ & - & - & - \\
\hline $\begin{array}{l}\text { MSM who used no } \mathrm{AB} \text { previous } \\
6 \text { months }\end{array}$ & $0 / 32(0.0)$ & - & - & - \\
\hline Neisseria bacilliformis & $1 / 96(1.0)$ & $2(-)$ & $0.125(-)$ & $1.5(-)$ \\
\hline Employees & $1 / 32(3.1)$ & $2(-)$ & $0.125(-)$ & $1.5(-)$ \\
\hline $\begin{array}{l}\text { MSM who used } A B \text { previous } \\
6 \text { months }\end{array}$ & $0 / 32(0.0)$ & - & - & - \\
\hline $\begin{array}{l}\text { MSM who used no } A B \text { previous } \\
6 \text { months }\end{array}$ & $0 / 32(0.0)$ & - & - & - \\
\hline
\end{tabular}


Table 2. Antimicrobial susceptibility of Neisseria isolates cultured from the oropharynx of 64 STI clinic attendees (men who have sex with men) and 32 employees of the Institute of Tropical Medicine (representing the general population) in Belgium. $A B$ antibiotics, $I Q R$ interquartile range, $M S M$ men who have sex with men, STI sexually transmitted infections.

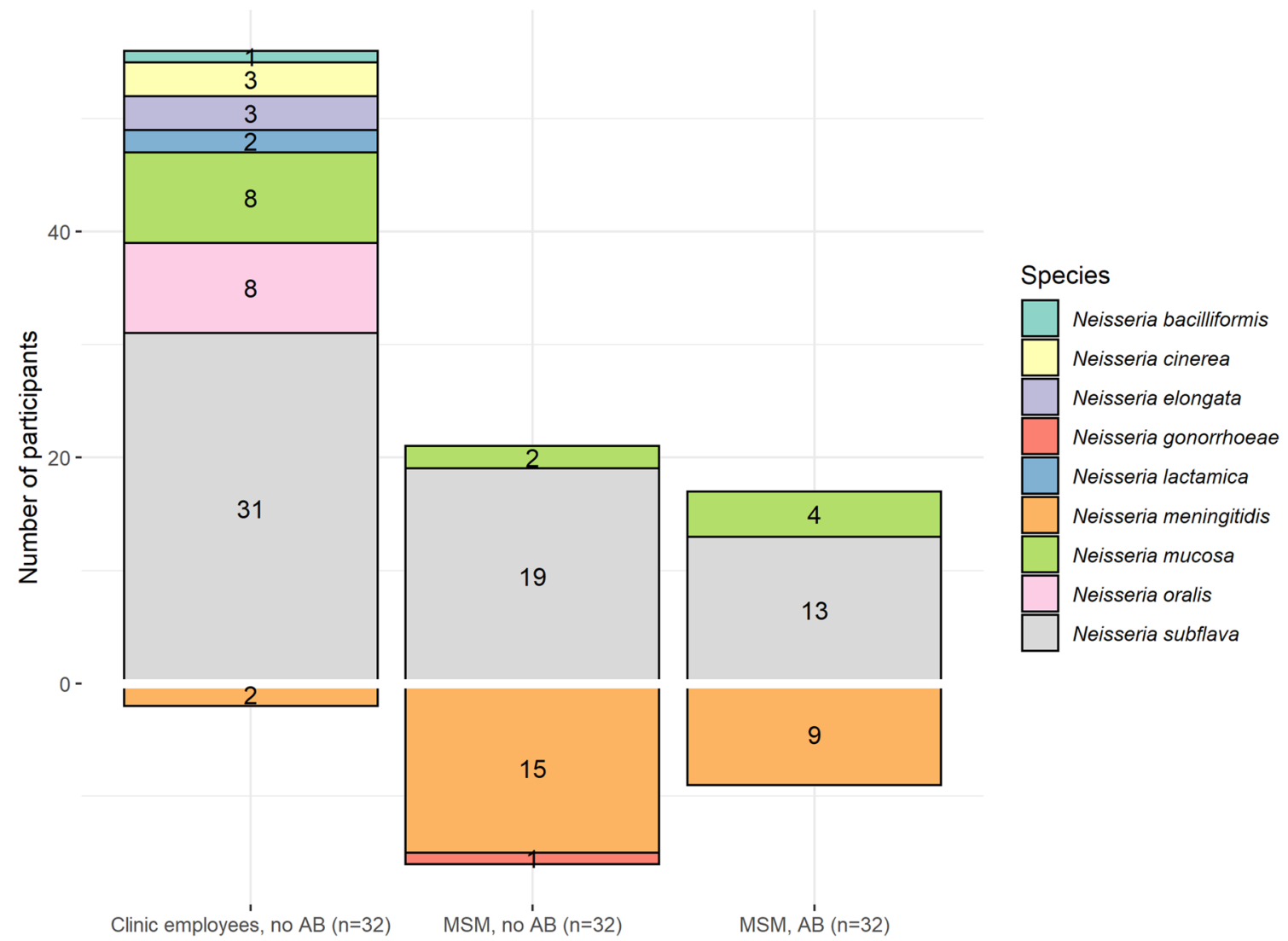

Figure 1. Prevalence and richness of Neisseria species, in absolute number of participants from whom the concerning species was isolated, per group. $A B$ antibiotics, MSM men who have sex with men.

elongata $(3 / 96,3.1 \%)$, N. lactamica $(2 / 96,2.1 \%)$, and $N$. bacilliformis $(1 / 96,1.0 \%)$. The pathogenic species were $N$. meningitidis (26/96, 27.1\% prevalence), and N. gonorrhoeae (one isolate from a MSM, 1.0\% prevalence).

The prevalence of non-pathogenic Neisseria was lower among MSM (51.6\%) than the employees $(100.0 \%$, $p<0.00001$, Table 2, Fig. 1), but for the pathogenic Neisseria this was the reverse: N. meningitidis was much more prevalent among MSM $(37.5 \%)$ than the employees $(6.3 \%, p<0.01)$.

MSM who used antimicrobials in the previous 6 months were less often colonised with N. meningitidis (28.1\%) than MSM who did not use antibiotics (46.9\%), but this difference was not statistically significant $(p=0.20)$.

Richness of non-pathogenic Neisseria species. Co-colonisation with multiple non-pathogenic Neisseria species was less common among MSM (7.8\% were colonised with two species) than the employees $(37.5 \%$ colonised with two species and $18.8 \%$ with three species).

In addition, while all seven non-pathogenic species were isolated from the employees, only two were isolated from MSM: N. subflava and N. mucosa. The richness of non-pathogenic species was thus lower among MSM (median of 1 species, IQR $0-1$ ) than the employees (median of 2 species, IQR $1-2, p<0.0001$ ).

Susceptibility of non-pathogenic Neisseria. The non-pathogenic Neisseria were significantly less susceptible (higher MICs) to all three antimicrobials than the pathogenic Neisseria $(p<0.0001$ for every antimicrobial, Table 2, Fig. 2). The non-pathogenic Neisseria isolated from MSM had significantly higher MICs for azithromycin (7.0 mg/L, IQR 3.0-280.2) and ciprofloxacin $(0.250 \mathrm{mg} / \mathrm{L}, \mathrm{IQR} 0.020-0.380)$ compared to those from the employees (3.0 mg/L, IQR 2.0-4.0, $p<0.0001$; and $0.023 \mathrm{mg} / \mathrm{L}, \mathrm{IQR} 0.012-0.064, p<0.001$, respectively; Table 2, Fig. 3). The MICs for ceftriaxone were similar in both groups (0.047 mg/L, IQR 0.032-0.084 in MSM versus 0.034 , IQR $0.026-0.064$ in the employees, $p=0.3$ ). There were no significant differences in MICs accord- 


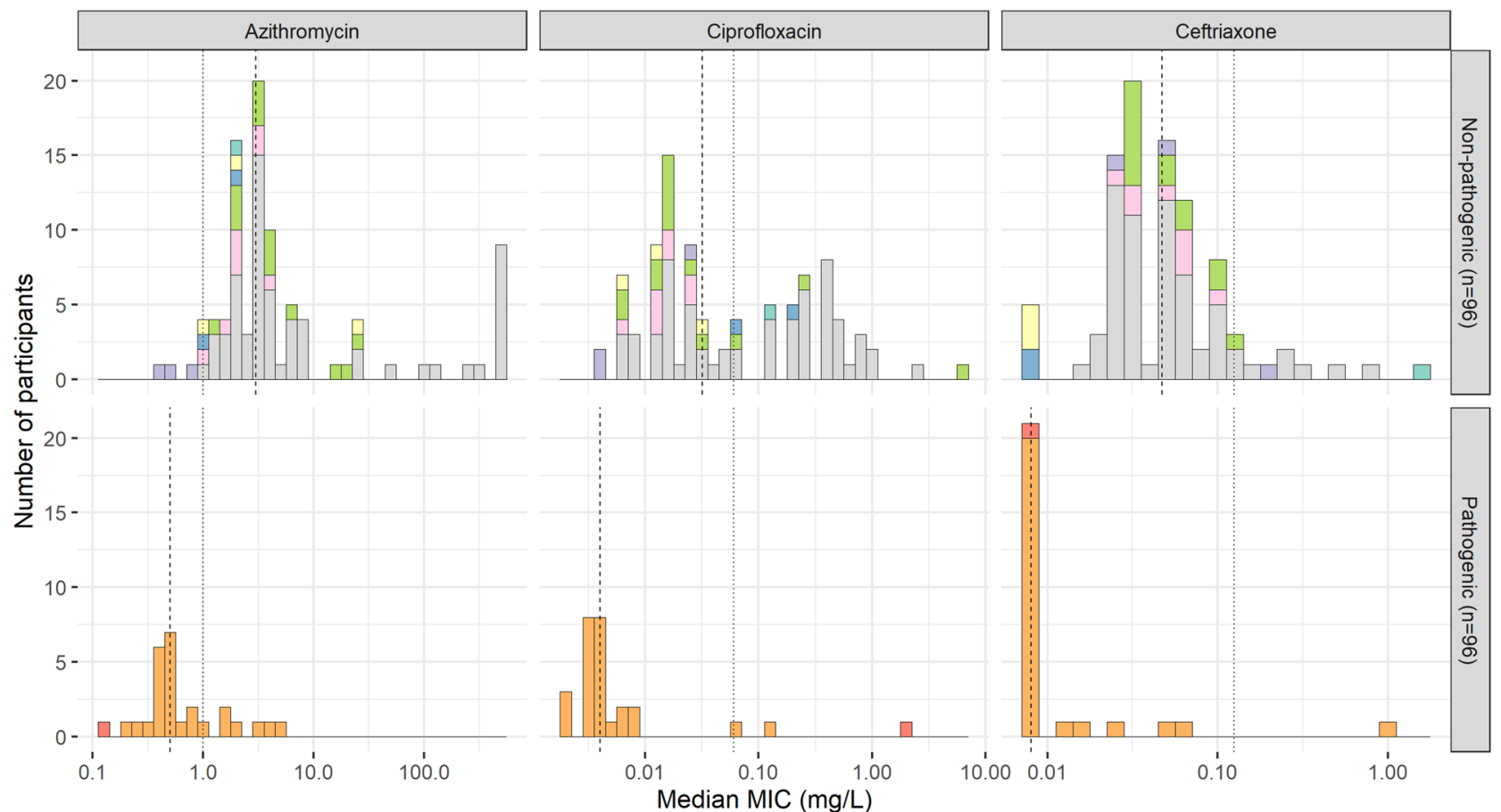

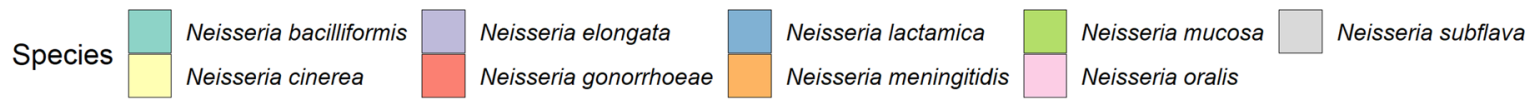

Figure 2. Minimum inhibitory concentration (MIC, mg/L) of pathogenic versus non-pathogenic Neisseria species isolated from all 96 participants. Numbers represent the number of participants with that specific median MIC per species. Vertical lines indicate the median of median MICs (dashed line) and the EUCAST v.11.0 cutoff for N. gonorrhoeae (dotted line) for each antibiotic.

ing to recent antimicrobial exposure in MSM. The stratified analysis for N. subflava showed similar findings. The stratified analysis for $N$. mucosa showed no significant differences in MICs between groups.

The sensitivity analysis based on a linear regression model confirmed the association between MSM and higher MICs for azithromycin (aOR 3.31, 95\% CI 1.42-7.72), but estimated an additional increase with recent antimicrobial use (aOR 2.99, 95\% CI 1.07-8.31).

For ciprofloxacin, the model suggested that the difference in MIC is only driven by higher MICs in those who were recently exposed to antimicrobials (aOR 3.79, 95\% CI 1.49-9.59, Table 3). In addition, the model estimated an association between MSM and higher MICs for ceftriaxone (aOR 1.58, 95\% CI 1.06-2.35).

Susceptibility of pathogenic Neisseria. For N. meningitidis, most isolates were highly susceptible to all three antimicrobials. According to current EUCAST breakpoints (v. 11.0), one isolate was resistant to ceftriaxone (MIC $1 \mathrm{mg} / \mathrm{L}$ ) and two participants had isolates with ciprofloxacin resistance (MIC 0.125 and $0.064 \mathrm{mg} / \mathrm{L}$ ).

The single N. gonorrhoeae isolate in this survey was susceptible to azithromycin (MIC $0.125 \mathrm{mg} / \mathrm{L}$ ) and ceftriaxone (MIC $<0.016 \mathrm{mg} / \mathrm{L}$ ) but resistant to ciprofloxacin (MIC $2 \mathrm{mg} / \mathrm{L}$ ).

\section{Discussion}

We found that contemporary oropharyngeal non-pathogenic Neisseria in MSM were less susceptible to antimicrobials than those from employees representing the general population. Recent antimicrobial exposure did not entirely explain the observed differences in susceptibility. This suggests that long-term participant- or populationlevel antimicrobial exposure plays an important role ${ }^{29}$. Indeed, MSM in PrEP programs consume a large amount of antimicrobials. One of the main drivers of excessive macrolide and cephalosporin consumption among PrEP users is the practice of screening asymptomatic MSM for gonorrhoea and chlamydia ${ }^{30}$. In some cohorts, macrolide consumption exceeds 12 defined daily doses per 1000 individuals per day (DID) ${ }^{30}$. This is multiple times what is consumed by typical general populations and is above the thresholds for inducing macrolide resistance in a range of bacterial species ${ }^{30,31}$. Reducing the intensity of screening for gonorrhoea and chlamydia among MSM may result in a four-fold decrease in macrolide consumption ${ }^{32}$.

Although lower than in MSM, the MICs of non-pathogenic Neisseria in the employees were considerably higher than in previous surveys. This is illustrated by N. subflava, the most prevalent species in our survey. A previous analysis of $N$. subflava isolates from the early 1980s found a considerably lower azithromycin MIC distribution (median $1.0 \mathrm{mg} / \mathrm{L}$, IQR $0.5-2.5 \mathrm{mg} / \mathrm{L}$ ) than that found in the current employees (median $3.0 \mathrm{mg} / \mathrm{L}$, IQR 2.3-4.0 mg/L ${ }^{16}$. 

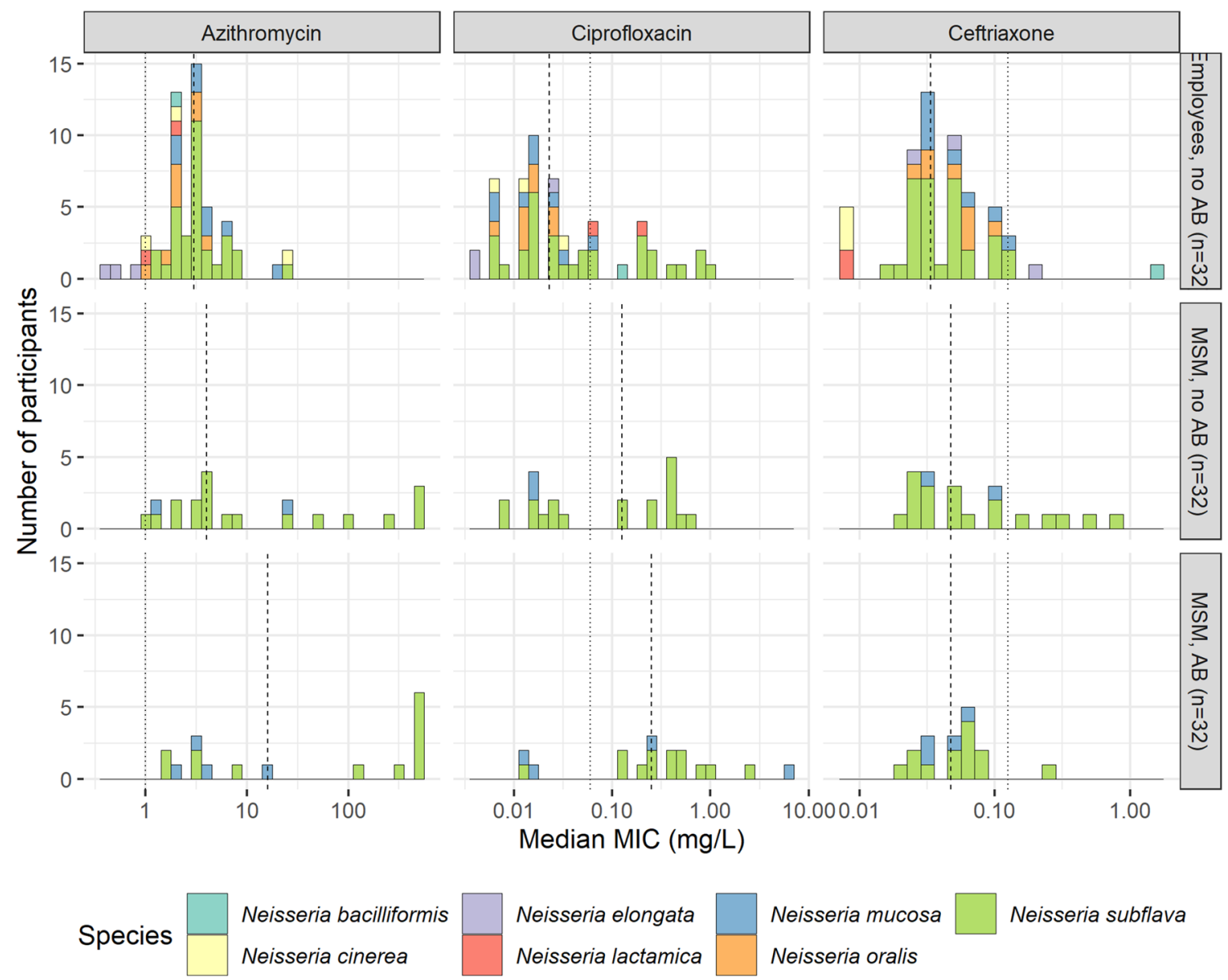

Figure 3. Minimum inhibitory concentration (MIC, $\mathrm{mg} / \mathrm{L}$ ) of non-pathogenic Neisseria species, per group. Numbers represent the number of participants with that specific median MIC per species. Vertical lines indicate the median of median MICs (dashed line) and the EUCAST v.11.0 cutoff for N. gonorrhoeae (dotted line) for each antibiotic.

\begin{tabular}{|c|c|c|c|c|c|c|c|}
\hline \multirow[b]{2}{*}{$\begin{array}{l}\text { All non-pathogenic } \\
\text { Neisseria }\end{array}$} & \multirow[b]{2}{*}{$\begin{array}{l}\text { Number of } \\
\text { participants (\%) }\end{array}$} & \multicolumn{2}{|l|}{ Ciprofloxacin } & \multicolumn{2}{|l|}{ Azithromycin } & \multicolumn{2}{|l|}{ Ceftriaxone } \\
\hline & & $\begin{array}{l}\text { Unadjusted OR } \\
(95 \% \text { CI })\end{array}$ & $\begin{array}{l}\text { Adjusted }{ }^{\mathrm{A}} \text { OR (95\% } \\
\text { CI) }\end{array}$ & $\begin{array}{l}\text { Unadjusted OR } \\
(95 \% \mathrm{CI})\end{array}$ & $\begin{array}{l}\text { Adjusted }^{\mathrm{A}} \text { OR }(95 \% \\
\text { CI) }\end{array}$ & $\begin{array}{l}\text { Unadjusted OR } \\
(95 \% \mathrm{CI})\end{array}$ & $\begin{array}{l}\text { Adjusted }^{A} \text { OR }(95 \% \\
\text { CI) }\end{array}$ \\
\hline \multicolumn{8}{|l|}{ Population } \\
\hline Employees & $32(33.3)$ & 1 (Ref) & 1 (Ref) & 1 (Ref) & 1 (Ref) & 1 (Ref) & 1 (Ref) \\
\hline MSM & $64(66.7)$ & $2.45(1.14-5.27)^{\star}$ & $1.69(0.78-3.66)$ & $4.38(1.97-9.77)^{*}$ & $3.31(1.42-7.72)^{*}$ & $1.66(1.05-2.61)^{*}$ & $1.58(1.06-2.35)^{\star}$ \\
\hline \multicolumn{8}{|c|}{ Used antibiotic in the previous 6 months } \\
\hline No & $64(66.7)$ & 1 (Ref) & 1 (Ref) & 1 (Ref) & 1 (Ref) & 1 (Ref) & 1 (Ref) \\
\hline Yes & $32(33.3)$ & $3.23(1.21-8.59)^{*}$ & $3.79(1.49-9.59)^{*}$ & $2.69(0.97-7.47)$ & $2.99(1.07-8.31)^{*}$ & $0.75(0.42-1.34)$ & $0.75(0.47-1.21)$ \\
\hline Neisseria subflava & $\begin{array}{l}\text { Number of } \\
\text { participants (\%) }\end{array}$ & Unadjusted & Adjusted $^{\mathrm{A}}$ & Unadjusted & Adjusted $^{\mathrm{A}}$ & Unadjusted & Adjusted $^{\mathrm{A}}$ \\
\hline \multicolumn{8}{|l|}{ Population } \\
\hline Employees & $31(49.2)$ & 1 (Ref) & NA & 1 (Ref) & NA & 1 (Ref) & NA \\
\hline MSM & $32(50.8)$ & $1.80(0.75-4.33)$ & NA & $4.07(1.51-10.95)^{\star}$ & NA & $1.68(1.06-2.67)^{\star}$ & NA \\
\hline \multicolumn{8}{|c|}{ Used antibiotic in the previous 6 months } \\
\hline No & $50(79.4)$ & $1(\mathrm{Ref})$ & NA & 1 (Ref) & NA & $1(\mathrm{Ref})$ & NA \\
\hline Yes & $13(20.6)$ & $3.34(1.13-9.86)^{*}$ & NA & $4.58(1.35-15.57)^{\star}$ & NA & $0.78(0.44-1.38)$ & NA \\
\hline
\end{tabular}

Table 3. Linear regression coefficients for change in geometric mean minimum inhibitory concentrations of non-pathogenic Neisseria for ciprofloxacin, azithromycin and ceftriaxone. CI Confidence Interval, MIC minimum inhibitory concentration, $N A$ not applicable, $O R$ odds ratio. ${ }^{\star}$ Estimate is statistically significant as the CI does not include $1 .{ }^{\text {A } A d j u s t e d ~ f o r ~ N e i s s e r i a ~ s p e c i e s . ~}$ 
In fact, the antimicrobial susceptibilities of the non-pathogenic Neisseria from the employees in our study were all higher than those from published reports from equivalent studies in the 1960s to the $1990 \mathrm{~s}^{33-37}$. Of note, the earliest survey of antimicrobial susceptibility in commensal Neisseria that we could locate, found that 28 clinical isolates of $N$. cinerea from Germany pre-1961 were highly susceptible to penicillin (MIC range $0.00015-0.0006 \mathrm{mg} / \mathrm{L})^{33}$. A likely explanation for this decrease in antimicrobial susceptibility over time is the level of antimicrobial consumption by the general Belgian population ${ }^{38}$. Macrolide consumption, for example, exceeded 3.0 DID in 2018 and 2019, which is well above a threshold of 1.3 DID which may select for resistance in pathogens like S. pneumoniae, M. genitalium, and T. pallidum ${ }^{31,39}$. Certain features of commensal bacteria suggest that such resistance threshold may even be lower for commensals than for pathogens. Thus, populationlevel antimicrobial consumption may have selected for circulating commensal Neisseria with elevated MICs ("Supplementary information").

The prevalence and richness of non-pathogenic Neisseria among MSM in our survey was lower than the employees and much lower than reported among MSM in Vietnam and the USA ${ }^{8,40}$. These low numbers among Belgian MSM taking PrEP could be explained by the high antimicrobial exposure of this population ${ }^{30}$. Similar to N. meningitidis, certain species of non-pathogenic Neisseria may be slower to acquire resistance to specific antimicrobials than other species ${ }^{9,13}$. For example, no isolates of $N$. elongata, $N$. lactamica or $N$. bacilliformis in our study had an azithromycin MIC greater than $2 \mathrm{mg} / \mathrm{L}$, whereas the median azithromycin MIC for N. subflava was $3 \mathrm{mg} / \mathrm{L}$ in the employees, $8 \mathrm{mg} / \mathrm{L}$ in MSM overall and $288 \mathrm{mg} / \mathrm{L}$ in the MSM group that had used antibiotics. This high-level resistance to azithromycin in N. subflava has been linked to the uptake of an $m s r D$ gene likely from oral streptococci ${ }^{41}$. Other Neisseria species have thus far not been found to be able to take up this gene or acquire such high-level resistance to azithromycin ${ }^{41}$. The higher consumption of antimicrobials in this MSM PrEP cohort could thus have eliminated the most susceptible non-pathogenic Neisseria species and thereby have reduced Neisseria species richness.

Conversely, the prevalence of N. meningitidis in our study was higher among MSM than the employees, which corroborates other reports of $N$. meningitidis prevalences up to $42.5 \%$ among $\mathrm{MSM}^{21-24}$. This exceeds by some margin the prevalence in young adults across the globe ${ }^{42} . N$. meningitidis is one of the most antimicrobial susceptible Neisseria species, as also observed in our current study ${ }^{43}$. A number of genetic differences between $N$. meningitidis and other Neisseria have been shown to underpin the reduced capacity of N. meningitidis to acquire resistance to various antimicrobials ${ }^{44,45}$.

Indeed, in our study, the prevalence of $N$. meningitidis in MSM exposed to antimicrobials was almost half that in unexposed MSM. The prevalence of N. meningitidis may thus temporarily decline due to the consumption of antimicrobials (as also shown in other studies ${ }^{21}$ ), but soon return to its equilibrium prevalence.

Several processes could explain the higher prevalence of N. meningitidis among MSM compared with members of the general population. One reason may be the high frequency of interpersonal contacts among MSM taking PrEP-like kissing and attending crowded night-clubs-during which transmission may occur ${ }^{21,46}$. Hypothetically, N. meningitidis may be more transmissible than non-pathogenic Neisseria and may thus outcompete the latter in recolonizing the pharynx after antimicrobial exposure. Lack of competition with other Neisseria species may be another explanation. A number of epidemiological, interventional and in-vitro studies have found evidence of such competition ${ }^{47}$. As an example, the presence of $N$. lactamica has been shown to be associated with a lower prevalence of $N$. meningitidis ${ }^{48-50}$.

If antibiotics reduced the prevalence of species such as N. lactamica in MSM, this may have left this population more susceptible to colonisation by $N$. meningitidis.

This study has a number of limitations, including the small sample sizes, single centre design and the fact that the samples were not representative of all MSM or the general Belgian population. Furthermore, two experimental factors of this survey may have caused underestimation of the richness of Neisseria species and the spectrum of their antibiotic susceptibilities. Firstly, the study depended on culturing Neisseria from the posterior oropharynx and tonsils. This design would likely have missed certain non-pathogenic Neisseria that preferentially inhabit other parts of the pharyn $\mathrm{x}^{51}$. Future studies could obtain samples by gargling with physiological saline to overcome this problem ${ }^{51}$. Secondly, only a minority of colonies grown on the agar plates were selected for species identification and MIC determination. We tried to pick at least one of each macroscopically distinct gram negative and oxidase positive colony per plate, but we may have missed particular Neisseria species with phenotypes similar to the sampled colonies. Metagenomic studies may also be a more sensitive way to profile the Neisseria microbiota and resistome than culture-based techniques. Finally, it would be instructive to repeat this study in settings with low population level antibiotic consumption.

In conclusion, we found high levels of resistance to azithromycin, ceftriaxone, and ciprofloxacin in oropharyngeal Neisseria among MSM and employees in Belgium. This finding is worrisome as non-pathogenic Neisseria provide a reservoir of resistance genes that can be readily transferred to pathogenic bacteria.

This AMR is most parsimoniously explained by excessive antibiotic exposure in the general Belgian population, but particularly in the MSM PrEP cohorts. Reduced screening for asymptomatic gonorrhoea and chlamydia may substantially reduce antimicrobial consumption by MSM.

The effect of such a policy change on the prevalence of AMR may be most easily demonstrated in the nonpathogenic Neisseria. Future studies may thus consider conducting regular surveys of antimicrobial susceptibility of non-pathogenic Neisseria in the general population and key populations such as MSM on PrEP as an early warning system of excessive antimicrobial consumption.

\section{Data availability}

All deidentified data are available as a Supplement to this manuscript. Additional related documents such as the study protocol, laboratory analysis plan, informed consent form can be obtained from the corresponding author upon reasonable request. 
Received: 8 October 2021; Accepted: 8 December 2021

Published online: 07 January 2022

\section{References}

1. Unemo, M. \& Shafer, W. M. Antimicrobial resistance in Neisseria gonorrhoeae in the 21st Century: Past, evolution, and future. Clin. Microbiol. Rev. 27, 587-613 (2014).

2. Chen, M. et al. Evolution of sequence type 4821 clonal complex hyperinvasive and quinolone-resistant meningococci. Emerg. Infect. Dis. 27, 1110-1122 (2021).

3. Zapun, A., Morlot, C. \& Taha, M. K. Resistance to $\beta$-lactams in Neisseria ssp due to chromosomally encoded penicillin-binding proteins. Antibiotics 5, 1-12 (2016).

4. Banhart, S. et al. The mosaic mtr locus as major genetic determinant of azithromycin resistance of Neisseria gonorrhoeae, Germany, 2018. J. Infect. Dis. https://doi.org/10.1093/infdis/jiab091 (2021).

5. Wadsworth, C. B., Arnold, B. J., Sater, M. R. A. A. \& Grad, Y. H. Azithromycin resistance through interspecific acquisition of an epistasis-dependent efflux pump component and transcriptional regulator in Neisseria gonorrhoeae. MBio 9, 1-17 (2018).

6. Hanao, M. et al. Molecular characterization of Neisseria gonorrhoeae isolates collected through a national surveillance programme in Japan, 2013: Evidence of the emergence of a ceftriaxone-resistant strain from a ceftriaxone-susceptible lineage. J. Antimicrob. Chemother. 76, 1769-1775 (2021).

7. Chen, M., Zhang, C., Zhang, X. \& Chen, M. Meningococcal quinolone resistance originated from several commensal neisseria species. Antimicrob. Agents Chemother. 64, e01494-19 (2020).

8. Dong, H. V. et al. Decreased cephalosporin susceptibility of oropharyngeal neisseria species in antibiotic-using men who have sex with men in Hanoi, Vietnam. Clin. Infect. Dis. 70, 1169-1175 (2020).

9. Fiore, M. A., Raisman, J. C., Wong, N. H., Hudson, A. O. \& Wadsworth, C. B. Exploration of the neisseria resistome reveals resistance mechanisms in commensals that may be acquired by N. Gonorrhoeae through horizontal gene transfer. Antibiotics $9,1-12$ (2020).

10. Manoharan-Basil, S. S. et al. Evidence of horizontal gene transfer of 50 S ribosomal genes rplB, rplD, and rplY in Neisseria gonorrhoeae. Front. Microbiol. 12, 1-17 (2021).

11. Gernert, K. M. et al. Azithromycin susceptibility of Neisseria gonorrhoeae in the USA in 2017: A genomic analysis of surveillance data. Lancet Microbe 1, e154-e164 (2020).

12. Kenyon, C., Laumen, J. \& Manoharan-Basil, S. Choosing new therapies for gonorrhoea: We need to consider the impact on the Pan-Neisseria Genome. A viewpoint. Antibiotics 10, 515 (2021).

13. Goytia, M., Thompson, S. T., Jordan, S. V. L. \& King, K. A. Antimicrobial resistance profiles of human commensal neisseria species. Antibiotics 10, 538 (2021).

14. Shen, Y. \& Chen, M. Prevalence, sequence type, and quinolone resistance of Neisseria lactamica carried in children younger than 15 years in Shanghai, China. J. Infect. 80, 61-68 (2020).

15. Takei, H. et al. Bacteriological analysis of Neisseria lactamica isolated from the respiratory tract in Japanese children. J. Infect. Chemother. 27, 65-69 (2021).

16. Laumen, J. G. E. et al. Markedly reduced azithromycin and ceftriaxone susceptibility in commensal neisseria species in clinical samples from belgian men who have sex with men. Clin. Infect. Dis. 72, 363-364 (2021).

17. Dong, H. V. et al. Reply to Laumen et al.. Clin. Infect. Dis. https://doi.org/10.1093/cid/ciaa568 (2020).

18. Kirkcaldy, R. D. et al. Neisseria gonorrhoeae antimicrobial resistance among men who have sex with men and men who have sex exclusively with women: The gonococcal isolate surveillance project, 2005-2010. Ann. Intern. Med. 158, 321-328 (2013).

19. Lewis, D. A. The role of core groups in the emergence and dissemination of antimicrobial-resistant N. gonorrhoeae. Sex. Transm. Infect. 89, iv47-iv51 (2013).

20. Kenyon, C. R. \& Schwartz, I. S. Effects of sexual network connectivity and antimicrobial drug use on antimicrobial resistance in neisseria gonorrhoeae. Emerg. Infect. Dis. 24, 1195-1203 (2018).

21. Ngai, S. et al. Carriage of neisseria meningitidis in men who have sex with men presenting to public sexual health clinics, New York City. Sex. Transm. Dis. 47, 541-548 (2020).

22. Tinggaard, M. et al. Oral and anal carriage of Neisseria meningitidis among sexually active HIV-infected men who have sex with men in Denmark 2014-2015. Int. J. Infect. Dis. 105, 337-344 (2021).

23. García, S. D. et al. Neisseria meningitidis aislada de muestras de hombres que tienen sexo con hombres. Rev. Argent. Microbiol. https://doi.org/10.1016/j.ram.2019.03.009 (2019).

24. Janda, W. M., Bohnhoff, M., Morello, J. A. \& Lerner, S. A. Prevalence and site-pathogen studies of Neisseria meningitidis and N. gonorrhoeae in Homosexual Men. JAMA J. Am. Med. Assoc. 244, 2060-2064 (1980).

25. Vuylsteke, B. et al. Daily and event-driven pre-exposure prophylaxis for men who have sex with men in Belgium : Results of a prospective cohort measuring adherence, sexual behaviour and STI incidence. J. Int. AIDS Soc. 22, 1-9 (2019).

26. Hoornenborg, E. et al. Sexual behaviour and incidence of HIV and sexually transmitted infections among men who have sex with men using daily and event-driven pre-exposure prophylaxis in AMPrEP: 2 year results from a demonstration study. Lancet HIV 6, e447-e455 (2019).

27. Van Dijck, C. et al. Antibacterial mouthwash to prevent sexually transmitted infections in men who have sex with men taking HIV pre-exposure prophylaxis (PReGo): A randomised, placebo-controlled, crossover trial. Lancet Infect. Dis. 3099, 657-667 (2021).

28. Bennett, J. S. et al. A genomic approach to bacterial taxonomy: An examination and proposed reclassification of species within the genus Neisseria. Microbiology 158, 1570-1580 (2012).

29. Olesen, S. W. et al. Azithromycin susceptibility among Neisseria gonorrhoeae isolates and seasonal macrolide use. J. Infect. Dis. 219, 619-623 (2019).

30. Kenyon, C., Baetselier, I. D. \& Wouters, K. Screening for STIs in PrEP cohorts results in high levels of antimicrobial consumption. Int. J. STD AIDS https://doi.org/10.1177/0956462420957519 (2020).

31. Kenyon, C., Manoharan-Basil, S. S. \& van Dijck, C. Is there a resistance-threshold for macrolide consumption? Positive evidence from an ecological analysis of resistance data from Streptococcus pneumoniae, Treponema pallidum and Mycoplasma genitalium. medRxiv 00, 10-12 (2020).

32. Vanbaelen, T. et al. Screening for STIs is one of the main drivers of macrolide consumption in PrEP users. Int. J. STD AIDS. 095646242110259 32(12), 1183-1184 (2021).

33. Berger, U. \& Paepcke, E. Untersuchungen über die asaccharolytischen Neisserien des menschlichen Nasopharynx. Zeitschrift für Hyg. und Infekt. 148, 269-281 (1962).

34. Sâez, J. A., Carmen, N. \& Vinde, M. A. Multicolonization of human nasopharynx due to Neisseria spp. Int. Microbiol. 1, 59-63 (1998).

35. Arreaza, L. What about antibiotic resistance in Neisseria lactamica?. J. Antimicrob. Chemother. 49, 545-547 (2002).

36. Karch, A., Vogel, U. \& Claus, H. Role of penA polymorphisms for penicillin susceptibility in Neisseria lactamica and Neisseria meningitidis. Int. J. Med. Microbiol. 305, 729-735 (2015).

37. Watanabe, Y., Takahashi, C., Ohya, H., Okazaki, N. \& Onoue, Y. Antibiotic susceptibility of Neisseria meningitidis from healthy and diseased persons in Japan. Kansenshogaku Zasshi 81, 669-674 (2007). 
38. Klein, E. Y. et al. Global increase and geographic convergence in antibiotic consumption between 2000 and 2015. Proc. Natl. Acad. Sci. USA 115, E3463-E3470 (2018).

39. ESAC. European Surveillance of Antimicrobial Consumption Program, Antimicrobial consumption database (ESAC-Net).

40. Knapp, J. S. \& Hook, E. W. Prevalence and persistence of Neisseria cinerea and other Neisseria spp. in adults. J. Clin. Microbiol. 26, 896-900 (1988).

41. de Block, T. et al. Wgs of commensal neisseria reveals acquisition of a new ribosomal protection protein (Msrd) as a possible explanation for high level azithromycin resistance in Belgium. Pathogens. 10, 384 (2021).

42. Peterson, M. E. et al. Serogroup-specific meningococcal carriage by age group: A systematic review and meta-analysis. BMJ Open 9, 1-9 (2019).

43. Diallo, K. et al. Pharyngeal carriage of Neisseria species in the African meningitis belt. J. Infect. 72, 667-677 (2016).

44. Antignac, A. et al. Correlation between alterations of the penicillin-binding protein 2 and modifications of the peptidoglycan structure in Neisseria meingitidis with reduced susceptibility to penicillin G. J. Biol. Chem. 278, 31529-31535 (2003).

45. Bash, M. C. \& Matthias, K. Antibiotic resistance in Neisseria. Antimicrob. Drug Resistance Clin. Epidemiol. Aspects. 2, 843 (2017).

46. Aral, S. O. Determinants of STD epidemics: Implications for phase appropriate intervention strategies. Sex. Transm. Infect. 78, i3-i13 (2002).

47. So, M. \& Rendón, M. A. Tribal warfare: Commensal Neisseria kill pathogen Neisseria gonorrhoeae using its DNA. Microb. Cell 6, 544-546 (2019).

48. Oliver, K. J. et al. Neisseria lactamica protects against experimental meningococcal infection. Infect. Immun. 70, 3621-3626 (2002).

49. Gold, R., Goldschneider, I., Lepow, M. L., Draper, T. F. \& Randolph, M. Carriage of neisseria meningitidis and neisseria lactamica in infants and children. J. Infect. Dis. 137, 112-121 (1978).

50. Deasy, A. M. et al. Nasal inoculation of the commensal Neisseria lactamica inhibits carriage of Neisseria meningitidis by young adults: A controlled human infection study. Clin. Infect. Dis. 60, 1512-1520 (2015).

51. Ando, N. et al. Modified self-obtained pooled sampling to screen for Chlamydia trachomatis and Neisseria gonorrhoeae infections in men who have sex with men. Sex. Transm. Infect. https://doi.org/10.1136/sextrans-2020-054666 (2020).

\section{Acknowledgements}

We want to acknowledge all survey participants for their kind participation.

\section{Author contributions}

C.K., S.A., E.B., I.D.B., J.L., C.V.D. and S.S.M.B. conceptualized the study. C.K. and C.V.D. collected the samples. S.A., J.L., I.D.B., D.M. and G.S. generated the laboratory results. J.L., C.V.D. and C.K. verified and analysed the data. C.V.D. and J.L. wrote the first draft of the manuscript. All authors reviewed and approved the final manuscript.

\section{Funding}

This study was funded by the Belgian Research Foundation - Flanders (FWO 121.00). The funder was not involved in any stage of the study.

\section{Competing interests}

The authors declare no competing interests.

\section{Additional information}

Supplementary Information The online version contains supplementary material available at https://doi.org/ 10.1038/s41598-021-03995-1.

Correspondence and requests for materials should be addressed to C.K.

Reprints and permissions information is available at www.nature.com/reprints.

Publisher's note Springer Nature remains neutral with regard to jurisdictional claims in published maps and institutional affiliations.

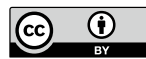

Open Access This article is licensed under a Creative Commons Attribution 4.0 International License, which permits use, sharing, adaptation, distribution and reproduction in any medium or format, as long as you give appropriate credit to the original author(s) and the source, provide a link to the Creative Commons licence, and indicate if changes were made. The images or other third party material in this article are included in the article's Creative Commons licence, unless indicated otherwise in a credit line to the material. If material is not included in the article's Creative Commons licence and your intended use is not permitted by statutory regulation or exceeds the permitted use, you will need to obtain permission directly from the copyright holder. To view a copy of this licence, visit http://creativecommons.org/licenses/by/4.0/.

(C) The Author(s) 2022 